\title{
Neuronal GLP1R mediates liraglutide's anorectic but not glucose-lowering effect
}

\author{
Stephanie Sisley, ${ }^{1,2}$ Ruth Gutierrez-Aguilar, ${ }^{3}$ Michael Scott, ${ }^{4}$ David A. D'Alessio, ${ }^{3}$ \\ Darleen A. Sandoval, ${ }^{3}$ and Randy J. Seeley ${ }^{3}$
}

\begin{abstract}
1Department of Pediatrics, Division of Endocrinology, Cincinnati Children's Hospital Medical Center, Cincinnati, Ohio, USA. 2Department of Pediatrics, Division of Nutrition, Baylor College of Medicine, Houston, Texas, USA. ${ }^{3}$ Department of Internal Medicine, Division of Endocrinology, University of Cincinnati College of Medicine, Cincinnati, Ohio, USA. ${ }^{4}$ Department of Pharmacology, University of Virginia School of Medicine, Charlottesville, Virginia, USA.
\end{abstract}

\begin{abstract}
Glucose control and weight loss are cornerstones of type 2 diabetes treatment. Currently, only glucagon-like peptide-1 (GLP1) analogs are able to achieve both weight loss and glucose tolerance. Both glucose and body weight are regulated by the brain, which contains GLP1 receptors (GLP1R). Even though the brain is poised to mediate the effects of GLP1 analogs, it remains unclear whether the glucose- and body weight-lowering effects of long-acting GLP1R agonists are via direct action on CNS GLP1R or the result of downstream activation of afferent neuronal GLP1R. We generated mice with either neuronal or visceral nerve-specific deletion of Glp1r and then administered liraglutide, a long-acting GLP1R agonist. We found that neither reduction of GLP1R in the CNS nor in the visceral nerves resulted in alterations in body weight or food intake in animals fed normal chow or a high-fat diet. Liraglutide treatment provided beneficial glucose-lowering effects in both chow- and high-fat-fed mice lacking GLP1R in the CNS or visceral nerves; however, liraglutide was ineffective at altering food intake, body weight, or causing a conditioned taste aversion in mice lacking neuronal GLP1R. These data indicate that neuronal GLP1Rs mediate body weight and anorectic effects of liraglutide, but are not required for glucose-lowering effects.
\end{abstract}

\section{Introduction}

Glucagon-like peptide-1 (GLP1) is a peptide produced both by intestinal enteroendocrine cells and a discrete population of hindbrain neurons. GLP1 acts through a G-protein-coupled receptor (GLP1R) expressed on pancreatic $\beta$ cells, CNS neurons, and peripheral neurons (including hepatic vagal afferents from the nodose ganglia) (1). GLP1R activation improves glucose tolerance and causes weight loss (2). Thus, long-acting GLP1 agonists are clinically used to control glucose and body weight in type 2 diabetic patients (3). Despite the popularity of these drugs, considerable controversy surrounds which GLP1Rs mediate their beneficial effects. Multiple publications have shown central GLP1R activation decreases food intake and improves glucose tolerance while inhibition increases food intake and worsens glucose tolerance (4). However, there is also evidence that activation of GLP1R expressed on peripheral vagal afferents can decrease food intake and improve glucose tolerance $(5,6)$. Despite these known effects of GLP1, DPP4 inhibitors, which increase the longevity of native GLP1, produce negligible weight loss (3). Thus, it is unknown how long-acting GLP1R agonists mediate their anorectic effects and whether they are mediated in a manner similar to their glucose-lowering effects.

\section{Results}

To discriminate between the effects of GLP1R expression on peripheral versus central nerves, we developed mouse models with selec-

Conflict of interest: David A. D’Alessio consults for Eli Lilly, Merck, Novo Nordisk, and Zealand. David A. D'Alessio receives research support from MannKind Pharmaceuticals, Sanofi-Aventis, and Ethicon Endo-Surgery. Darleen A. Sandoval serves on the scientific advisory board at Ethicon Endo-Surgery. Darleen A. Sandoval receives research support from Ethicon Endo-Surgery, Boehringer Ingelheim, and Novo Nordisk. Randy J. Seeley is a paid speaker for Novo Nordisk and Merck. Randy J. Seeley serves on scientific advisory boards for Novo Nordisk, Novartis, Angiochem, Zealand, Takeda, Eli Lilly, Boehringer Ingelheim, Eisai, and Forest Pharmaceuticals. Randy J. Seeley receives research support from Novo Nordisk, Ethicon Endo-Surgery, Ablaris, Boehringer Ingelheim, Givaudan, and Zealand. Randy J. Seeley has equity in Zafgen. Citation for this article: J Clin Invest. 2014;124(6):2456-2463. doi:10.1172/JCI72434 tive reduction of key GLP1R-expressing components of the central or peripheral nervous system. This was accomplished through the insertion of loxP sites surrounding exons 6 and 7 of the Glp1r gene (Figure 1A) and subsequent breeding to either nestin-Cre (7) or Phox 2b-Cre (8) mice, respectively. Nestin is an intermediate filament protein expressed in neuroepithelial cells that, when used as a promoter for Cre expression, specifically disrupts the gene of interest in the brain $(7,9,10)$. Mice with site-specific knockdown (KD) of the GLP1R in the CNS (nestin-Cre Glp1rflox/flox, herein referred to as GLP1R KD $\left.{ }^{\Delta \text { Nestin}}\right)$ showed markedly decreased Glp1r mRNA in the hypothalamus and brainstem compared with control mice, but no reduction in the nodose ganglion or lung (Supplemental Figure 1A; supplemental material available online with this article; doi:10.1172/JCI72434DS1). We also observed a decrease in pancreatic Glp1r expression (Supplemental Figure 1A). However, while some data show expression of nestin in pancreatic progenitor cells (11), it has not been shown to be present in endocrine cells (12). The gene for Glp1r is located in the nodose ganglia, a cell body of the vagus nerve, and the protein is found within neurons innervating the hepatic portal vein (13). Phox $2 b$ is a homeobox gene expressed in the autonomic nervous system, including the nucleus of the solitary tract and dorsomotor nucleus of the vagus (14). Thus, using a Cre-recombinase driven by a Phox $2 b$ promotor, we generated mice with site-specific KD of the GLP1R in visceral afferent and efferent nerves (Phox 2b-Cre Glp1 flox/flox, herein referred to as GLP1R $\mathrm{KD}^{\Delta \text { Phox } 2 \mathrm{~b}}$ ). Accordingly, GLP1R $\mathrm{KD}^{\Delta \mathrm{Phox} 2 \mathrm{~b}}$ had decreased expression of the Glp1r in the nodose ganglion, but no change in the hypothalamus or hindbrain (Supplemental Figure 1B).

Since endogenous GLP1 has been hypothesized to regulate food intake and body weight (6), we tested the role of CNS and vagal GLP1Rs in the regulation of food intake and body weight. In chow and high-fat diet conditions, body weight did not differ among GLP1R KD ${ }^{\Delta \text { Nestin }}$, GLP1R KD ${ }^{\Delta \text { Phox2b }}$, or controls (Figure 1, B and D). Consistent with this, no differences existed in body composition (Figure 1, C and E). Although no differences existed in food intake 

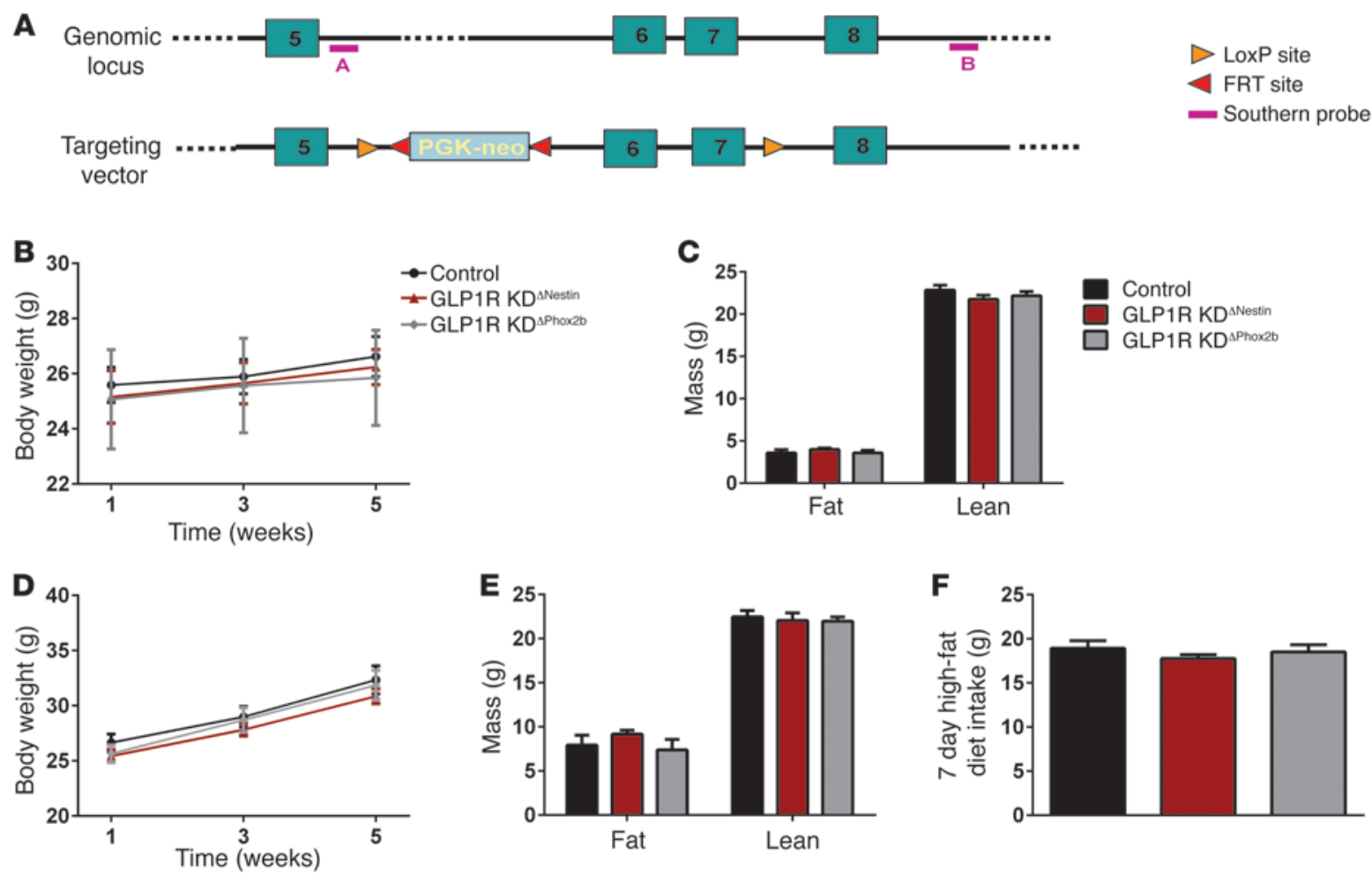

Figure 1

Characterization of GLP1R KD ${ }^{\Delta N e s t i n}$ and GLP1R KD ${ }^{\Delta P h o x 2 b}$ mice. (A) Glp1r floxed construct. (B) Body weight analysis of chow-fed Glp1r mutant mice. (C) Body composition analysis on chow diet. (D) Body weight analysis of high-fat-fed Glp1r mutant mice. (E) Body composition analysis after 7 weeks of high-fat diet. (F) Seven-day high-fat diet intake. Statistical analysis was with 2-way ANOVA (B-E) or 1-way ANOVA (F) with Tukey's post-hoc. Black, controls; red, GLP1R KD ${ }^{\Delta \text { Nestin; gray, GLP1R KD }}{ }^{\Delta \text { Phox2b. }}$

among the groups on high-fat diet (Figure 1F), we have observed conflicting data regarding the food intake of chow-fed GLP1R $\mathrm{KD}^{\Delta \text { Nestin }}$ mice. In separate cohorts of GLP1R KD ${ }^{\Delta \text { Nestin }}$ mice, we have seen either similar or increased food intakes when compared with controls (Supplemental Figure 2, A and B, respectively). These discrepancies will be the subject of future investigations. However, given the high degree of GLP1R KD in both strains of mice, these data suggest that CNS and vagal GLP1Rs are not necessary for the normal regulation of food intake or body weight.

Despite these data, long-acting GLP1R agonists are well documented as having potent food intake-reducing effects. Prior research has pointed to a role for central GLP1 signaling in the anorectic effects of long-acting GLP1R agonists, but the respective contributions of central versus vagal GLP1R is unclear. Thus, a crucial question is whether such agonists act directly in the CNS or on vagal afferents to mediate these effects. In response to an acute s.c. injection of liraglutide, control and GLP1R KD ${ }^{\Delta P h o x 2 b}$ chow-fed mice had significantly decreased food intake at 4 and 24 hours (Figure 2, A and B). However, acute liraglutide administration caused a modest reduction in food intake at 4 hours but not at 24 hours in GLP1R KD ${ }^{\Delta \text { Nestin }}$ mice (Figure 2, A and B). In a new cohort of high-fat-fed GLP1R ${ }^{\text {Nestin }}$ mice, we repeated this study and found that liraglutide was able to decrease food intake compared with saline, but this response was attenuated compared with that of controls (Figure 2, C and D). Thus, CNS but not vagal GLP1Rs are necessary for the full acute anorectic response to a long-acting GLP1R agonist.
Acute administration of either GLP1 or long-acting GLP1R agonists causes visceral illness, which is reflected in rodent models by a pronounced conditioned taste aversion $(\mathrm{CTA})(15,16)$. Whether this effect is the result of activation of GLP1Rs in the CNS or a secondary result of reduced gastric emptying is controversial. Thus, we next tested whether liraglutide caused a CTA in our mouse models. Both chow-fed control and GLP1R KD ${ }^{\Delta \mathrm{Phox} 2 \mathrm{~b}}$ mice developed a CTA to a novel flavor paired with liraglutide. However, the pairing had no effect in GLP1R KD ${ }^{\Delta \text { Nestin }}$ mice, despite the ability to develop a CTA to lithium chloride (Figure 2E). Combined with our previous data, these results highlight the importance of CNS GLP1R in the acute anorectic effects of a long-acting GLP1R agonist.

Chronic administration of liraglutide continues to cause weight loss long after attenuation of its aversive effects (15). Thus, to understand the mechanism underlying weight loss associated with chronic administration of a long-acting GLP1R agonist (17), we administered liraglutide or saline daily for 15 days in these mouse models after 5 weeks of high-fat feeding. GLP1R KD ${ }^{\Delta N e s t i n}$ mice treated with liraglutide lost more weight than saline controls, but significantly less weight than liraglutide-treated GLP1R KD ${ }^{\Delta P h o x 2 b}$ or control mice (Figure 3, A and B). The body weight loss was proportional to the food intake changes observed with chronic liraglutide treatment (Figure 3C). Over the last 7 days of treatment, liraglutide decreased food intake in GLP1R KD ${ }^{\Delta \text { Phox } 2 b}$ and control mice, but not in GLP1R KD ${ }^{\Delta \text { Nestin }}$ mice (Figure 3D). Liraglutide treatment also significantly decreased fat mass in both control 
A

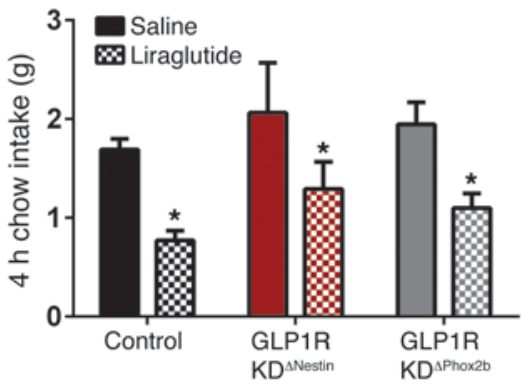

C

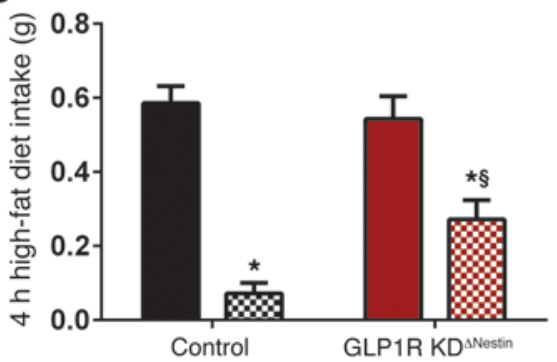

B

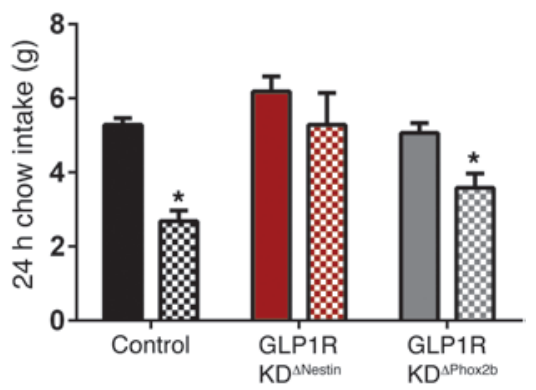

D

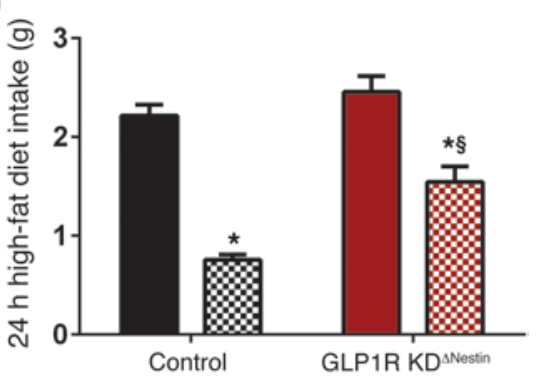

Figure 2

Acute effects of liraglutide in GLP1R KD ${ }^{\Delta \text { Nestin }}$ and GLP1R KD ${ }^{\triangle P h o x 2 b}$ mice. (A and B) Anorectic effects of liraglutide at $4(A)$ and 24 (B) hours in chow-fed mice. (C and D) Anorectic effects of liraglutide in GLP1R KD ${ }^{\Delta \text { Nestin }}$ high-fat-fed mice at 4 (C) and 24 (D) hours. (E) CTA test after acute liraglutide administration in chow-fed mice. Statistical analysis was with 2-way ANOVA with repeated measures ( $\mathbf{A}$ and $\mathbf{B})$ or without repeated measures (C-E) with Tukey's post-hoc. ${ }^{*} P<0.05$ vs. same genotype; ${ }^{\S} P<0.05$ vs. same drug, different genotype

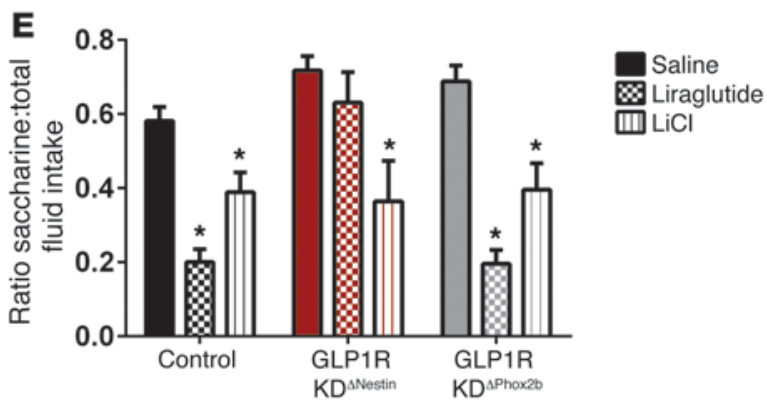

and GLP1R KD ${ }^{\Delta \text { Phox2b }}$, mice but not in GLP1R KD ${ }^{\Delta \text { Nestin }}$ mice (Figure $3 \mathrm{E})$. There were no significant differences in lean mass across groups or treatments (Figure $3 \mathrm{~F}$ ). Together, these data show that CNS but not vagal GLP1R are necessary for the chronic anorectic and weight-lowering effects of liraglutide.

Although GLP1 has been widely hypothesized to lower glucose by increasing $\beta$ cell insulin secretion, other data implicate vagal and CNS GLP1R contributions $(13,18)$. We performed both oral and i.p. glucose tolerance tests (GTTs) to assess the role of the central and vagal CNS GLP1R in endogenous GLP1 signaling. GLP1R $\mathrm{KD}^{\Delta \text { Nestin }}$ mice had no differences in oral or i.p. glucose tolerance compared with controls whether studied under chow- or high-fatfed conditions (Figure 4, A-I). The chow-fed oral GTT was run in a separate cohort of mice without the availability of concurrent analysis of GLP1R KD ${ }^{\Delta \text { Phox2b }}$ mice. GLP1R KD ${ }^{\Delta \text { Phox2b }}$ did not show any differences in glucose tolerance in the chow-fed i.p. GTT or high-fat diet fed i.p. and oral GTTs (Figure 4, A, B, and E-I). During the i.p. GTT on high-fat-fed mice, there was no interaction of genotype on insulin secretion at baseline or at 15 minutes (Figure 4F). Homeostasis model assessment estimated insulin resistance (HOMA-IR) calculations, a measure of insulin resistance, also found no differences at baseline, nor were there differences in the insulin times glucose product at the 15 -minute time point (data not shown). Thus, these data imply that neither CNS nor vagal GLP1R are necessary for the physiological regulation of glucose.
Given the previously published studies on the effects of GLP1 in the brain on glucose homeostasis $(1,13)$, it is reasonable to hypothesize that the actions of long-acting GLP1 agonists to improve glucose regulation are not limited to their direct actions on $\beta$ cells. Thus, we next tested the necessity of CNS or vagal GLP1R to meditate the glucose-lowering effects of liraglutide. Liraglutide caused significant decreases in glucose excursions in all genotypes during i.p. GTTs after both acute and chronic administration (Figure 5, A and B). There was no effect of genotype on insulin levels during an i.p. GTT after 14 days of liraglutide (measured at 0 and 15 minutes after i.p. glucose) (Figure 5C). These data demonstrate that neither vagal nor CNS GLP1R are necessary for the glucoselowering effects of liraglutide and suggest that these effects are also independent of insulin concentrations.

\section{Discussion}

These data are the first, to our knowledge, to identify tissue-specific effects of CNS GLP1Rs on body weight and glucose regulation. This information is critical to understanding both the physiological relevance of the GLP1 system and the mechanism of action of GLP1 analogs. Given the high degree of tissue-specific GLP1R KD in both strains of our mice, our data suggest that CNS and vagal GLP1Rs are not necessary for the normal regulation of food intake or body weight. This stands in contrast to other approaches showing decreased food intake with direct CNS GLP1R agonism (19) 

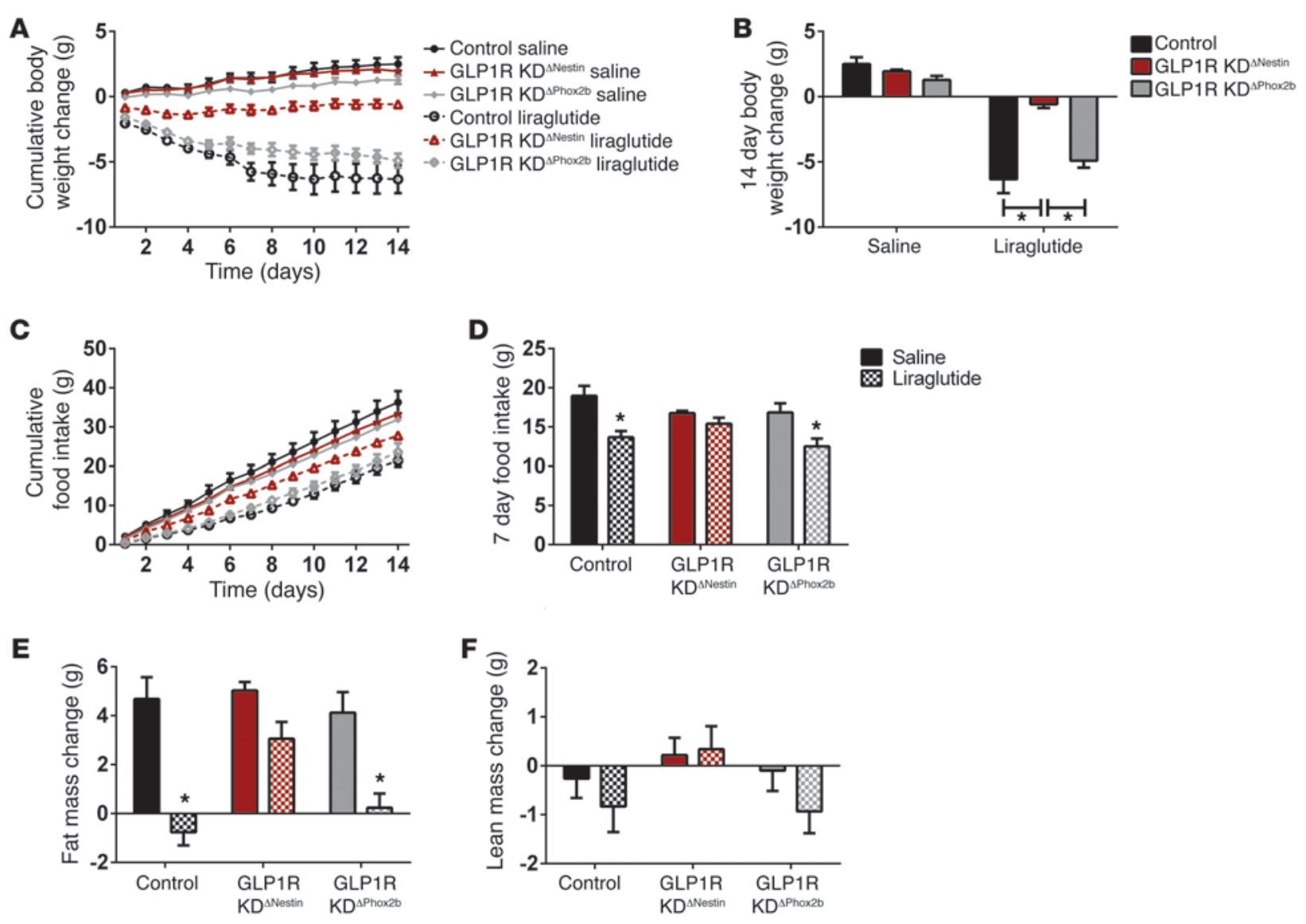

\section{Figure 3}

Liraglutide requires CNS GLP1R for anorectic and weight-lowering effects in high-fat-fed mice. (A) Longitudinal body weights of G/p1r mutant mice treated with a long-acting GLP1R agonist or saline. (B) Cumulative weight loss of 14 days. (C) Longitudinal 14-day cumulative food intake of mice. (D) Total food intake during last 7 days of treatment. (E) Fourteen-day fat-mass change of mice. (F) Fourteen-day lean-mass change in mice. Black, controls; red, GLP1R KD ${ }^{\Delta N e s t i n}$; gray, GLP1R KD ${ }^{\triangle P h o x 2 b ; ~ s o l i d, ~ s a l i n e ~ t r e a t m e n t ; ~ p a t t e r n e d, ~ l i r a g l u t i d e ~ t r e a t m e n t . ~ S t a t i s t i c a l ~ a n a l y s i s ~}$ was with 2-way ANOVA with Tukey's post-hoc with repeated measures (A and $\mathbf{C})$ or without repeated measures $(\mathbf{B}$ and $\mathbf{D}-\mathbf{F})$. ${ }^{*} P<0.05$ compared with saline treatment, same genotype unless otherwise indicated.

or increased food intake with CNS GLP1R antagonism or KD (6). However, our data complement findings from whole-body Glp1r male $\mathrm{KO}$ mice, which have limited differences in food intake or body weight $(20,21)$. We do note that there may be a role for CNS GLP1R signaling in the regulation of food intake, since we have found differing results across multiple cohorts of mice. Importantly, we have never observed a difference in body weight or body fat. This implies that either these alterations in food intake are highly transient or compensated by via changes in energy expenditure. Future work will need to address this issue.

Previous data on the role of the vagus in GLP1 signaling is controversial, with some showing decreased GLP1/GLP1R agonist-mediated anorexia after vagotomy $(5,22,23)$ and others demonstrating no effect of vagotomy in GLP1-mediated anorexia $(15,24)$. Interestingly, we find that GLP1Rs in the nodose ganglia of the vagus nerve are not necessary for the anorectic effects of a long-acting GLP1R agonist. This is the only approach that specifically targets the GLP1R on the vagus nerve without altering vagal afferent or efferent signaling activated by other substances. Thus, this approach gives more conclusive evidence that long-acting GLP1R agonists do not require substantial vagal GLP1R signaling for their anorectic effects.
Recent evidence indicates that the anorexic effects of exendin-4 are mediated entirely by the GLP1R (25). Here, we found that GLP1R KD ${ }^{\Delta \text { Nestin }}$ are highly resistant to the food intake and body weight lowering effects of liraglutide, supporting an important role for CNS GLP1R in mediating these effects. It should be noted that Cre driven by the nestin promoter has been widely used for loss-of-function of specific genes in the CNS, but it is expressed in other tissues, as evidenced by the reduced GLP1R in the pancreas of GLP1R KD ${ }^{\Delta \text { Nestin }}$ animals $(11,12)$. However, it is unlikely that other tissues targeted by nestin are likely to make large contributions to the effects of GLP1 analogs on these behavioral end points.

We and others have shown previously that CNS pharmacological blockade of the GLP1R increases food intake $(19,26)$ and that central GLP1Rs are important in the anorectic effects of peripheral liraglutide (5). Additionally, there are published data showing that liraglutide can cross the blood-brain barrier (27). Interestingly, on a chow diet, we saw no acute effect of liraglutide on food intake in GLP1R KD Nestin animals, but on a high-fat diet we saw an attenuated effect at 24 hours. It is possible that this is a dose-dependent response, since liraglutide is dosed on body weight and thus highfat fed animals received a higher dose. This attenuated effect on 

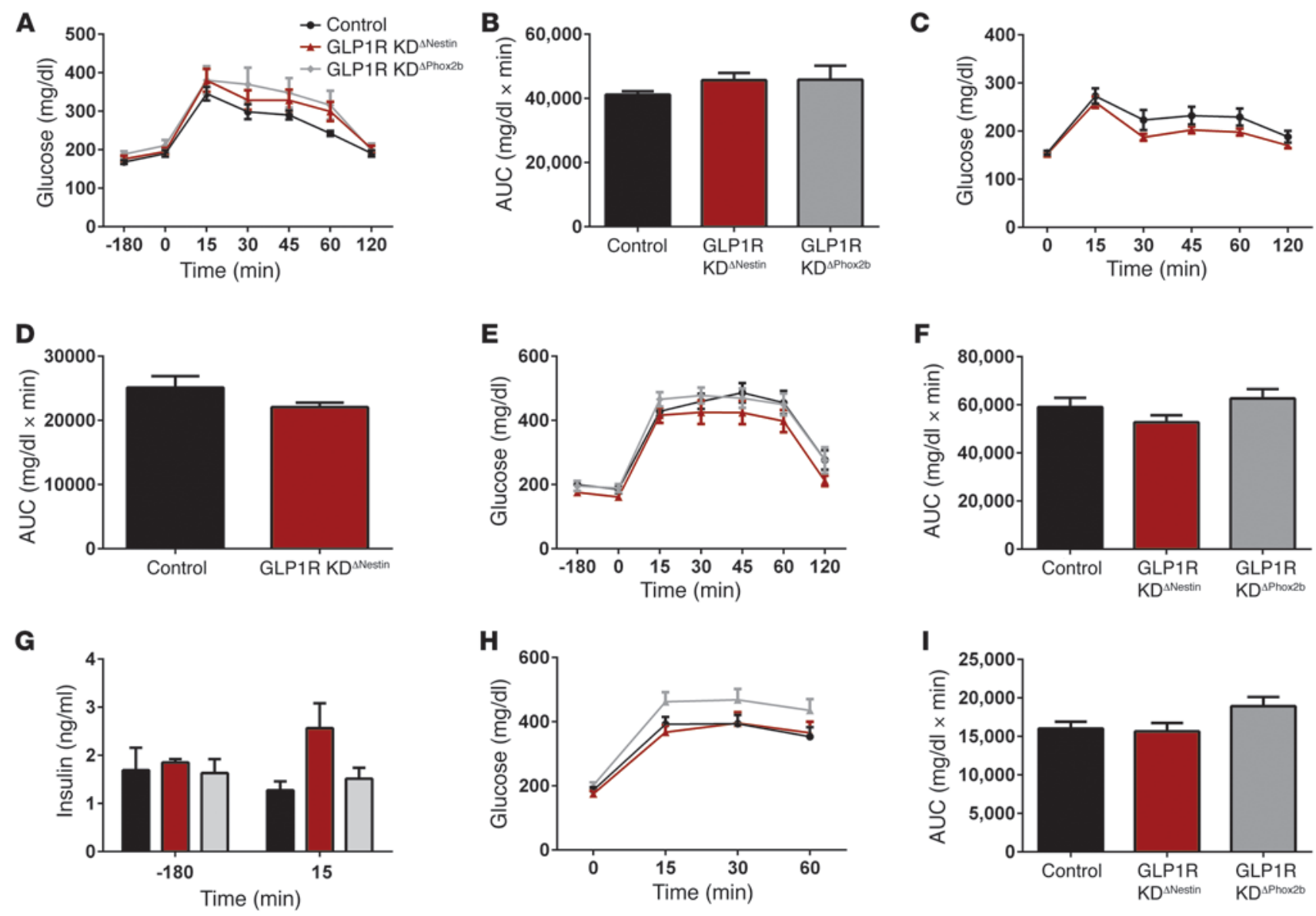

\section{Figure 4}

Glucose tolerance phenotypes of Glp1r mutant mice. (A) i.p. GTT on chow diet. (B) AUC for A. (C) Oral GTT on chow diet. Main effect of genotype. (D) AUC for C. (E) i.p. GTT on high-fat diet. (F) AUC for E. (G) Insulin response to i.p. glucose. (H) Oral GTT on high-fat diet. (I) AUC for H. Statistical analysis was with 2-way ANOVA with repeated measures (A, C, E, G, and H) or 1-way ANOVA (B, D, F, and I) with Tukey's post-hoc.

food intake was apparent during chronic liraglutide treatment as well. The decrease in body weight after liraglutide treatment mirrors the decrease in food intake in the GLP1R KD ${ }^{\Delta \text { Nestin }}$ mice. Thus, it is likely that the body weight effects of GLP1R agonists are driven by their effects on food intake. To put it in numbers, in GLP1R KD ${ }^{\Delta \text { Nestin }}$ animals, liraglutide treatment resulted in 24.76 fewer kcal ingested and $2.26 \mathrm{~g}$ less body fat than saline treatment. The $2.26 \mathrm{~g}$ body fat would equate to $20.38 \mathrm{kcal}$ in intake, meaning there are only $4.38 \mathrm{kcal}$ unaccounted for in the 14-day treatment course. This could be a result of inherent measurement inaccuracy in the food intake; however, we cannot exclude small differences in energy expenditure. Additionally, recent clinical literature demonstrates that weight loss secondary to GLP1R agonists can be explained via alterations in energy intake without effects on energy expenditure $(28,29)$. Regardless of their limited role in normal body weight, these data do point to an important role for CNS GLP1Rs in mediating food intake, body weight, and body fat responses to a long-acting GLP1R agonist.

Interestingly, these data do not implicate CNS or vagal GLP1Rs in the glucose-lowering effects of a long-acting GLP1R agonist. Previous data suggested that the hindbrain was important for GLP1's glucose-lowering effects $(6,30,31)$. Reduced expression of preproglucagon in the hindbrain resulted in worsened glucose tolerance in high-fat-fed rats (6), but this may have been secondary to weight gain. The portal vein, which is innervated by the nodose ganglia and robustly expresses GLP1R, has also been hypothesized to contribute to endogenous GLP1's actions (13). Local administration of GLP1R antagonists in the portal vein reduced normal glucose tolerance more than the same dose delivered into the general circulation (13). Furthermore, systemic GLP1R mediation of glycemic control has been shown to require vagal afferent fibers (23). Conversely, a blood-brain barrier-impermeable GLP1 analog increased neuronal activation in key hindbrain regions and lowered glucose excursions without an effect on body weight $(30,31)$. Importantly, the current approach specifically reduces GLP1R signaling in vagal afferents without altering the integrity of other vagal afferent or efferent signals. Thus, in our model, important elements of glucose regulation such as gastric emptying are still intact (32). Our data conflict with published reports showing an improvement in glucose tolerance and muscle glucose utilization after intracerebroventricular GLP1R antagonism with exendin 9-39 (33). However, this report used a high-fat (72\%), carbohydrate-free diet, which is very different from the diet used in these studies, making direct comparisons difficult.

The current data do not find an effect of a GLP1R agonist on insulin secretion. This result comes with important caveats. It 
A

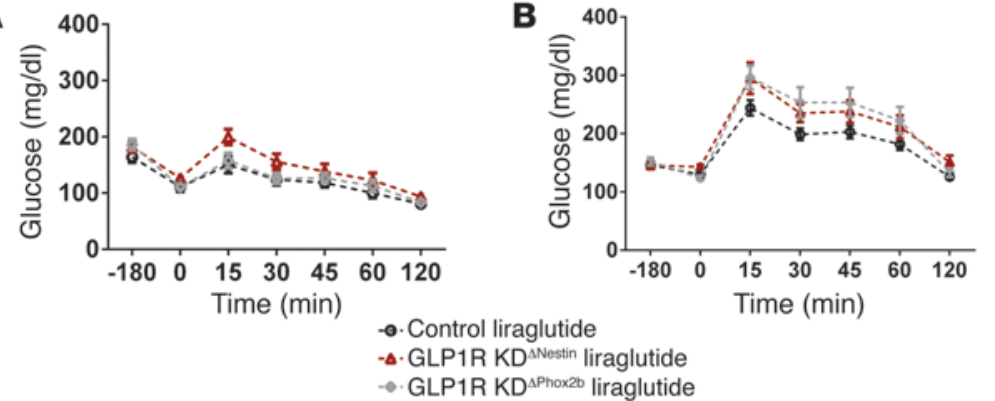

C

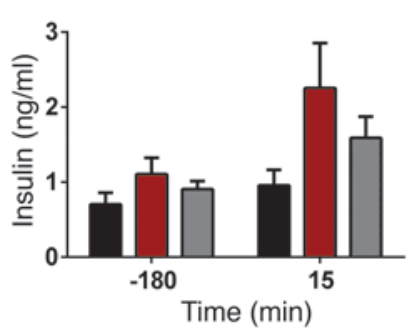

Figure 5

Liraglutide lowers glucose tolerance despite KD of central GLP1 receptors. (A) i.p. GTT on chow diet. (B) i.p. GTT on high-fat diet after 14 days of chronic liraglutide (from Figure 3A). (C) Insulin level during the GTT in B. Statistical analysis was with 2-way ANOVA with repeated measures with Tukey post-hoc.

is possible that 15 minutes may simply have not been the ideal time point to see the impact of liraglutide on insulin secretion and therefore this limits our ability to assess the impact of the GLP1R KD. Nevertheless, our data show that neither CNS nor vagal GLP1R are necessary for the ability of a long-acting GLP1R agonist to improve glucose tolerance.

In conclusion, these data highlight the important differences between the physiological effects of the endogenous GLP1 system and GLP1-based therapies. CNS and vagal GLP1Rs are not necessary for normal food intake, body weight regulation, or normal glucose tolerance. Vagal GLP1Rs also have little impact on GLP1R agonist-induced regulation of food intake and body weight. However, CNS GLP1Rs are necessary for the food intake, weight loss effects, and CTA effects of a long-acting GLP1R agonist. Identifying the key receptors for the effects of GLP1-based therapies holds promise for understanding the differences that exist in the clinical actions of long-acting GLP1 agonists and DPP-4 inhibitors (3) as well as designing more effective therapies that target key aspects of the GLP1 system to increase their effectiveness. Most importantly, these data show the importance of CNS action of antidiabetic therapies in order to also promote weight loss in diabetic patients.

\section{Methods}

Animals. Male mice were singly housed and maintained on a 12-hour light/12-hour dark cycle with food ad libitum, unless otherwise noted. Animals were initially placed on chow diet (Harlan Teklad no. 7012; 3.1 $\mathrm{kcal} / \mathrm{g}, 25 \%$ protein, $17 \%$ fat, $58 \%$ carbohydrates per $\mathrm{kcal}$ ), and studies were started after 8 weeks of age using age-matched mice. Mice received acute food intake studies and an i.p. GTT and were then placed on a high-fat, high-sucrose diet (Research Diets no. D12331; $5.56 \mathrm{kcal} / \mathrm{gm}, 16 \%$ protein, $58 \%$ fat, 26\% carbohydrates per kcal) for 4 weeks (age range 13-25 weeks, average age 16 weeks). Mice received an oral GTT after 4 weeks of high-fat feeding, recovered for an additional week, and then underwent a chronic 2-week study. A separate cohort of male GLP1R KD $\mathrm{KNestin}^{\Delta \text { and control mice }}$ was generated for additional studies. These studies are evident by the lack of concurrent GLP1R KD ${ }^{\triangle \mathrm{Phox} 2 \mathrm{~b}}$ data.

Generation of Glp1r conditional KO mice. A Glp1r conditional KO mouse was generated by introducing a loxP site on either side of exons 6 and 7 of the Glp1r gene (ref. 34 and Figure 1A). The Glp1r targeting vector was electroporated into 129 ES cells. Targeted Glp1r ES clones were identified by Southern blotting. Positive ES clones were injected into blastocysts to generate chimera by the ES core at the University of Cincinnati. Germ- line transmission was verified in agouti mice, and then these mice were bred to Flp-recombinase mice (Jackson Laboratory, stock number 003800; C57BL/6) to remove the neomycin resistance cassette. Subsequently, these mice were bred to $\mathrm{C} 57 \mathrm{BL} / 6$ mice to remove the Flp gene before breeding to Cre mice. We generated neuronal KD of the GLP1R by breeding the floxed mice with nestin-Cre mice (Jackson Laboratories, no. 003771) (35) and nodose-specific KD by breeding to Phox2b-Cre mice (a gift from Joel Elmquist, University of Texas Southwestern, Dallas, Texas, USA) (36).

Breeding. Mice heterozygous for both the Cre (nestin or Phox 2b) and Glp1r floxed gene were bred together to produce mice with Cre-specific loss of the Glp1r gene and littermate controls. We used only male nestin-Cre mice for breeding and performed DNA copy number analysis (TaqMan Copy Number Assays; Applied Biosciences) to verify the absence of the transmission of a germline deletion $(7,37)$ that is common in females and less frequent ( $10 \%$ in our studies) in males. Initially, 4 different control groups

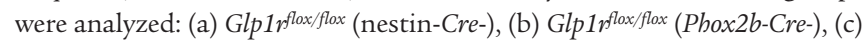
nestin-Cre Glp1 $r^{f l o x /+}$, and (d) Phox $2 b$-Cre Glp1 $f^{f o x /+}$. No measure revealed differences among these different control genotypes (Supplemental Figure 3). Consequently, for additional statistical power and ease of presentation, we pooled their results and refer to this group collectively as controls.

Validation. To validate proper gene targeting, mice were sacrificed by $\mathrm{CO}_{2}$ asphyxiation and tissues (brain, lung, liver, pancreas, nodose ganglion) were rapidly removed and frozen. Tissue RNA was extracted using a QIAGEN RNeasy kit. cDNA was isolated, and real-time quantitative PCR (qPCR) was performed using a TaqMan 7900 Sequence Detection System with TaqMan Universal PCR Master Mix and TaqMan Gene Expression Assays (all from Applied Biosystems). Relative mRNA expression for the Glp1r using a primer/probe targeted to exons 6 and 7 of the Glp1r gene (Mm00470859_cn; Applied Biosystems) was calculated relative to L32 using the $\Delta \Delta C T$ method (38).

Food intake studies. For all acute food intake studies, mice were fasted 4 hours prior to their dark cycle in order to increase the sensitivity of detecting anorectic responses without causing compensatory hyperphagia (39). During acute food intake studies, liraglutide (Novo Nordisk; $400 \mu \mathrm{g} / \mathrm{kg}$ ) or saline $(10 \mathrm{ml} / \mathrm{kg})$ was injected s.c. beginning 1 hour prior to the dark cycle in a counterbalanced approach. This dose was based on advisement by Novo Nordisk and is in line with previously published effective doses. Food was measured at baseline and 1, 2, 4, and 24 hours after lights out. Body weights were measured at baseline and at 24 hours. A crossover study was performed the following week and the results pooled.

CTA test. Weight-randomized mice were deprived of water for 24 hours and then given access to $0.15 \%$ of saccharin solution (presented on the opposite side of the normal location of the water bottle) for 2 hours. They 
then received saline, liraglutide, or $\mathrm{LiCl}(0.15 \mathrm{M}$ in a volume equivalent to $2 \%$ of each animal's body weight, s.c.); this was followed by removal of the saccharin bottle and replacement of the water bottle. The dose of liraglutide was approximately $400 \mu \mathrm{g} / \mathrm{kg}$. The following day, animals were water deprived. Twenty-four hours later, water and saccharin bottles were returned to their previous locations. Fluid intake was measured 24 hours after access to the bottles.

GTT. During the acute i.p. GTT, mice were fasted for 4 hours. At $t=-120$ minutes, mice were given liraglutide $(400 \mu \mathrm{g} / \mathrm{kg})$ or saline $(10 \mathrm{ml} / \mathrm{kg})$ s.c. At $t=0$ minutes, mice were given an i.p. injection of $1.5 \mathrm{~g} / \mathrm{kg} 25 \%$ dextrose. Blood glucose was measured by tail blood sampling at $-180,0,15,30$, 45,60 , and 120 minutes. $40 \mu \mathrm{l}$ blood was collected from a subset of mice ( $n=5 /$ group) into heparinized tubes at 180 prior to and 15 minutes after glucose administration and immediately placed on ice. An additional i.p. GTT was performed at the conclusion of the chronic study as above, except mice were given liraglutide $(1000 \mu \mathrm{g} / \mathrm{kg})$ or saline s.c. An oral GTT was performed as above for the acute i.p. GTT, except a flat dose of $50 \mathrm{mg}(\sim 1.6$ $\mathrm{g} / \mathrm{kg}$ ) D25W glucose gavage (40) was given at $t=0$ minutes.

Insulin analysis. Serum collected from a GTT was analyzed using an ultrasensitive mouse insulin ELISA kit (Crystal Chem) per the manufacturer's protocol for a low-range assay.

Chronic study. Male diet-induced obese mice were randomized by weight and assigned to receive either liraglutide or saline for 15 days. Mice were injected at least 1 hour prior to the dark cycle with liraglutide as follows: $300 \mu \mathrm{g} / \mathrm{kg}$ on day $0,600 \mu \mathrm{g} / \mathrm{kg}$ on day 1 , and $1000 \mu \mathrm{g} / \mathrm{kg}$ on days 2 to 16 . All injections were given s.c. as a $10 \mathrm{ml} / \mathrm{kg}$ bolus. 24-hour food intake measurements and body weights were performed daily. On day 14, a GTT was performed as above.

1. Sandoval D. CNS GLP-1 regulation of peripheral glucose homeostasis. Physiol Behav. 2008;94(5):670-674.

2. Buse JB, et al. Exenatide once weekly versus liraglutide once daily in patients with type 2 diabetes (DURATION-6): a randomised, open-label study. Lancet. 2013;381(9861):117-124.

3. Niswender K, et al. Weight change with liraglutide and comparator therapies: an analysis of seven phase 3 trials from the liraglutide diabetes development programme. Diabetes Obes Metab. 2013;15(1):42-54.

4. Lockie SH. The GLP-1 receptor in the brain: role in neuroendocrine control of energy metabolism and treatment target for obesity. J Neuroendocrinol. 2013;25(7):597-604

5. Kanoski SE, Fortin SM, Arnold M, Grill HJ, Hayes MR. Peripheral and central GLP-1 receptor populations mediate the anorectic effects of peripherally administered GLP-1 receptor agonists, liraglutide and exendin-4. Endocrinology. 2011;152(8):3103-3112.

6. Barrera JG, Jones KR, Herman JP, D'Alessio DA, Woods SC, Seeley RJ. Hyperphagia and increased fat accumulation in two models of chronic CNS glucagon-like peptide-1 loss of function. J Neurosci. 2011;31(10):3904-3913.

7. Tronche F, et al. Disruption of the glucocorticoid receptor gene in the nervous system results in reduced anxiety. Nat Genet. 1999;23(1):99-103.

8. Scott MM, Williams KW, Rossi J, Lee CE, Elmquist JK. Leptin receptor expression in hindbrain GLP-1 neurons regulates food intake and energy balance in mice. J Clin Invest. 2011;121(6):2413-2421.

9. Bruning JC, et al. Role of brain insulin receptor in control of body weight and reproduction. Science. 2000;289(5487):2122-2125.

10. Dubois NC, Hofmann D, Kaloulis K, Bishop JM, Trumpp A. Nestin-Cre transgenic mouse line NesCre1 mediates highly efficient Cre/loxP mediated recombination in the nervous system, kidney, and somite-derived tissues. Genesis. 2006;44(8):355-360.

11. Dorisetty RK, Kiran SG, Umrani MR, Boindala S, Bhonde RR, Venkatesan V. Immuolocalization of
Body composition analysis. Mice underwent body composition analysis (EchoMRI) prior to placement on high-fat diet and on day 16 of the chronic study.

Statistics. Statistical analyses were performed with Prism (GraphPad Software v6). Statistical tests performed are included in each figure legend. Data are presented as mean \pm SEM. Statistical significance was set at $P<0.05$ for all analyses. Number of animals for each experiment is listed in Supplemental Methods.

Study approval. All studies were approved by the University of Cincinnati Institutional Animal Care and Use Committee.

\section{Acknowledgments}

We would like to acknowledge the superior technical assistance of Bailing Li, Joyce Sorrell, Emily Matter, Alfor Lewis, Eric Smith, and April Haller. We would also like to thank Joel Elmquist (University of Texas Southwestern) for making the Phox $2 \mathrm{~b}$-cre mice available for these experiments. These studies were funded by the following NIH grants: 1F32DK091077-01A1 (to S. Sisley); DK057900 (to D.A. D'Alessio); DK082480 (to D.A. D'Alessio); DK093848 (to R.J. Seeley) and DK082480 (to D.A. Sandoval). R.J. Seeley and D.A. D'Alessio also received funding from Novo Nordisk.

Received for publication July 29, 2013, and accepted in revised form February 27, 2014.

Address correspondence to: Stephanie Sisley, 1100 Bates St., Houston, Texas 77030, USA. Phone: 713.798.0391; Fax: 713.798.7057; E-mail: Stephanie.sisley@bcm.edu. nestin in pancreatic tissue of mice at different ages. World J Gastroenterol. 2008;14(46):7112-716.

12. Delacour A, Nepote V, Trumpp A, Herrera PL. Nestin expression in pancreatic exocrine cell lineages. Mech Dev. 2004;121(1):3-14.

13. Vahl TP, et al. Glucagon-like peptide-1 (GLP-1) receptors expressed on nerve terminals in the portal vein mediate the effects of endogenous GLP-1 on glucose tolerance in rats. Endocrinology. 2007; 148(10):4965-4973.

14. Pattyn A, Morin X, Cremer H, Goridis C, Brunet JF. Expression and interactions of the two closely related homeobox genes Phox2a and Phox2b during neurogenesis. Development. 1997;124(20):4065-4075.

15. Kanoski SE, Rupprecht LE, Fortin SM, De Jonghe $\mathrm{BC}$, Hayes MR. The role of nausea in food intake and body weight suppression by peripheral GLP-1 receptor agonists, exendin-4 and liraglutide. Neuropharmacology. 2012;62(5-6):1916-1927.

16. Kinzig KP, D’Alessio DA, Seeley RJ. The diverse roles of specific GLP-1 receptors in the control of food intake and the response to visceral illness. J Neurosci. 2002;22(23):10470-10476.

17. Larsen PJ, Fledelius C, Knudsen LB, Tang-Christensen M. Systemic administration of the longacting GLP-1 derivative NN2211 induces lasting and reversible weight loss in both normal and obese rats. Diabetes. 2001;50(11):2530-2539.

18. Sandoval DA, Bagnol D, Woods SC, D'Alessio DA, Seeley RJ. Arcuate glucagon-like peptide 1 receptors regulate glucose homeostasis but not food intake. Diabetes. 2008;57(8):2046-2054.

19. Barrera JG, D’Alessio DA, Drucker DJ, Woods SC, Seeley RJ. Differences in the central anorectic effects of glucagon-like peptide- 1 and exendin- 4 in rats. Diabetes. 2009;58(12):2820-2827.

20. Scrocchi LA, et al. Glucose intolerance but normal satiety in mice with a null mutation in the glucagon-like peptide 1 receptor gene. Nat Med. 1996; 2(11):1254-1258.

21. Ayala JE, Bracy DP, James FD, Burmeister MA, Was- serman DH, Drucker DJ. Glucagon-like peptide-1 receptor knockout mice are protected from highfat diet-induced insulin resistance. Endocrinology. 2010;151(10):4678-4687.

22. Hunt JV, Washington MC, Sayegh AI. Exenatide and feeding: possible peripheral neuronal pathways. Peptides. 2012;33(2):285-290.

23. Hayes MR, et al. The common hepatic branch of the vagus is not required to mediate the glycemic and food intake suppressive effects of glucagonlike-peptide-1. Am J Physiol Regul Integr Comp Physiol. 2011;301(5):R1479-R1485.

24. Zhang J, Ritter RC. Circulating GLP-1 and CCK-8 reduce food intake by capsaicin-insensitive, nonvagal mechanisms. Am J Physiol Regul Integr Comp Physiol. 2012;302(2):R264-R273.

25. Tatarkiewicz K, Sablan EJ, Polizzi CJ, Villescaz C, Parkes DG. Long-term metabolic benefits of exenatide in mice are mediated solely via the known glucagon-like peptide 1 receptor [published online ahead of print: January 29, 2014]. Am J Physiol Regul Integr Comp Physiol. doi:10.1152/ajpregu.00495.2013.

26. Alhadeff AL, Rupprecht LE, Hayes MR. GLP-1 neurons in the nucleus of the solitary tract project directly to the ventral tegmental area and nucleus accumbens to control for food intake. Endocrinology. 2012;153(2):647-658.

27. Hunter K, Hölscher C. Drugs developed to treat diabetes, liraglutide and lixisenatide, cross the blood brain barrier and enhance neurogenesis. BMC Neurosci. 2012;13:33.

28. van Can J, Sloth B, Jensen CB, Flint A, Blaak EE, Saris WH. Effects of the once-daily GLP-1 analog liraglutide on gastric emptying, glycemic parameters, appetite and energy metabolism in obese, nondiabetic adults [published online ahead of print September 3, 2013]. Int J Obes (Lond). doi:10.1038/ ijo.2013.162

29. Tan TM, et al. Coadministration of glucagon-like peptide-1 during glucagon infusion in humans results in increased energy expenditure and amelioration of 
hyperglycemia. Diabetes. 2013;62(4):1131-1138.

30. Kim JG, et al. Development and characterization of a glucagon-like peptide 1-albumin conjugate: the ability to activate the glucagon-like peptide 1 receptor in vivo. Diabetes. 2003;52(3):751-759.

31. Yamamoto H, et al. Glucagon-like peptide-1 receptor stimulation increases blood pressure and heart rate and activates autonomic regulatory neurons. J Clin Invest. 2002;110(1):43-52.

32. Thompson DG, Wingate DL, Thomas M, Harrison D. Gastric emptying as a determinant of the oral glucose tolerance test. Gastroenterology. 1982;82(1):51-55.

33. Knauf C, et al. Brain glucagon-like peptide 1 signaling controls the onset of high-fat diet-induced insulin resistance and reduces energy expenditure.
Endocrinology. 2008;149(10):4768-4777.

34. Wilson-Pérez HE, et al. Vertical sleeve gastrectomy is effective in two genetic mouse models of glucagon-like Peptide 1 receptor deficiency. Diabetes. 2013;62(7):2380-2385.

35. Belgardt BF, et al. Hypothalamic and pituitary c-Jun $\mathrm{N}$-terminal kinase 1 signaling coordinately regulates glucose metabolism. Proc Natl Acad Sci U S A. 2010;107(13):6028-6033

36. Rossi J, et al. Melanocortin- 4 receptors expressed by cholinergic neurons regulate energy balance and glucose homeostasis. Cell Metab. 2011;13(2):195-204.

37. Trumpp A, Depew MJ, Rubenstein JL, Bishop JM, Martin GR. Cre-mediated gene inactivation demonstrates that FGF8 is required for cell survival and patterning of the first branchial arch. Genes Dev. 1999;13(23):3136-3148.

38. Livak KJ, Schmittgen TD. Analysis of relative gene expression data using real-time quantitative PCR and the 2(-Delta Delta C(T)) Method. Methods. 2001;25(4):402-408.

39. Ellacott KLJ, Morton GJ, Woods SC, Tso P, Schwartz MW. Assessment of feeding behavior in laboratory mice. Cell Metab. 2010;12(1):10-17.

40. McGuinness OP, Ayala JE, Laughlin MR, Wasserman DH. NIH experiment in centralized mouse phenotyping: the Vanderbilt experience and recommendations for evaluating glucose homeostasis in the mouse. AmJ Physiol Endocrinol Metab. 2009;297(4):E849-E855 